\title{
Functional Coupling of the Amygdala in Depressed Patients Treated with Antidepressant Medication
}

\author{
Chi-Hua Chen', John Suckling', Cinly Ooi', Cynthia HY Fu' ${ }^{2}$, Steve CR Williams ${ }^{3}$, Nicholas D Walsh', \\ Martina T Mitterschiffthaler ${ }^{3}$, Emilio Merlo Pich ${ }^{4}$ and Ed Bullmore*, ${ }^{*, 1,5}$
}

'Department of Psychiatry, Brain Mapping Unit, University of Cambridge, Addenbrooke's Hospital, Cambridge, UK; ${ }^{2}$ MRC Social, Genetic and Developmental Psychiatry Centre, Institute of Psychiatry, King's College, London, UK; ${ }^{3}$ Centre for Neuroimaging Science, Institute of Psychiatry, King's College, London, UK; ${ }^{4}$ Psychiatry Centre for Excellence in Drug Discovery, Clinical Pharmacology and Discovery Medicine, GlaxoSmithKline SpA, Verona, Italy; ${ }^{5}$ Clinical Unit Cambridge, Clinical Pharmacology and Discovery Medicine, GlaxoSmithKline R\&D, Cambridge, UK

\begin{abstract}
The amygdala plays a central role in various aspects of affect processing and mood regulation by its rich anatomical connections to other limbic and cortical regions. It is plausible that depressive disorders, and response to antidepressant drugs, may reflect changes in the physiological coupling between the amygdala and other components of affect-related large-scale brain systems. We explored this hypothesis by mapping the functional coupling of right and left amygdalae in functional magnetic resonance imaging data acquired from 19 patients with major depressive disorder and 19 healthy volunteers, each scanned twice (at baseline and 8 weeks later) during performance of an implicit facial affect processing task. Between scanning sessions, the patients received treatment with an antidepressant drug, fluoxetine $20 \mathrm{mg} /$ day. We found that the amygdala was positively coupled bilaterally with medial temporal and ventral occipital regions, and negatively coupled with the anterior cingulate cortex. Antidepressant treatment was associated with significantly increased coupling between the amygdala and right frontal and cingulate cortex, striatum, and thalamus. Treatment-related increases in functional coupling to frontal and other regions were greater for the left amygdala than for the right amygdala. These results indicate that antidepressant drug effects can be measured in terms of altered coupling between components of cortico-limbic systems and that these effects were most clearly demonstrated by enhanced functional coupling of the left amygdala.
\end{abstract}

Neuropsychopharmacology (2008) 33, 1909-1918; doi:I0.1038/sj.npp.1301593; published online 7 November 2007

Keywords: amygdala; connectivity; regression; systems; pharmacodynamic; biomarker

\section{INTRODUCTION}

The dominant emotional symptoms in major depression include depressed mood, anhedonia, anxiety, and irritability (American Psychiatric Association, 1994). Amygdala dysfunction may have implications for the pathogenesis of these depressive symptoms (Drevets, 2001, 2003). It is known that the amygdala has a role in the recall of emotional memories, for instance those acquired in fearful or anxious contexts (LaBar and Cabeza, 2006); and excessive amygdalar activity seen in depressed patients is arguably related to their tendency to 'ruminate' on emotionally aversive memories (Drevets, 2001). The amygdala is also involved in the perception of emotional cues (LeDoux, 2000) and the acquisition of emotional memory (LaBar and Cabeza, 2006). Depressed patients may have abnormally elevated secretion of both noradrenaline (Wong

\footnotetext{
*Correspondence: Professor E Bullmore, Department of Psychiatry, Brain Mapping Unit, University of Cambridge, Addenbrooke's Hospital, Cambridge CB2 2QQ, UK, Tel: + 4401223 336582, Fax: + 4401223 33658।, E-mail: etb23@cam.ac.uk

Received 16 March 2007; revised 6 September 2007; accepted 7 September 2007
}

et al, 2000) and cortisol (Lopez et al, 1999), which generally have an acute effect of memory enhancement (LaBar and Cabeza, 2006); therefore, together with the presence of amygdala overactivity, the chance that emotionally neutral stimuli are perceived or remembered as being aversive may increase (Drevets, 2001). Restoration of abnormal amygdala activity (Sheline et al, 2001), noradrenaline metabolite levels (Potter et al, 1985), and stress hormone regulation (Barden, 2004) were reported in depressed patients after antidepressant treatment, which may be associated with the amelioration of some depressive symptoms. Thus amygdala function in an abnormal endocrine milieu has been invoked to provide a mechanism for the negative bias that is typical of the mnemonic and perceptual functions of patients with depression.

However, understanding the pathophysiology of the amygdala must take into account the richness of its anatomical connections with other regions (Aggleton, 1993). Nonhuman primate studies provide valuable insights into a functional understanding of the anatomical connectivity of the amygdala. The key functions of the amygdala are: firstly, processing emotionally valent sensory input signals and emotional memories by interacting with other regions such as sensory cortices, thalamus, and hippocampus; secondly, 
expressing emotions by connecting with central autonomic structures (Ghashghaei and Barbas, 2002; Barbas, 2000). It follows that functionally abnormal connectivity of the amygdala with other brain regions could be fundamental to many of the wide range of autonomic, emotional, and cognitive symptoms associated with the pathological state of depressive disorder.

Most previous functional neuroimaging studies of depressive disorder have measured brain activation at each region or voxel of the images and tested for a difference in local brain function between depressed patients and appropriate comparison subjects. In this way, abnormalities of amygdala activation have been quite consistently demonstrated in depression (Drevets et al, 1992, 2002b; Sheline et al, 2001; Siegle et al, 2002). The same methodological approach has also been used to demonstrate local changes in amygdala function associated with antidepressant treatment (Drevets et al, 2002a; Fu et al, 2004; Sheline et al, 2001).

However, despite the theoretical expectation that aspects of the depressive syndrome might emerge from abnormal interactions between the amygdala and other brain regions, relatively few functional neuroimaging studies have adopted a multivariate approach to data analysis required to detect the effects of antidepressant drugs on neural connectivity (Anand et al, 2005a, b; Irwin et al, 2004; Seminowicz et al, 2004).

On the basis of these data and the more general theoretical considerations outlined above, we hypothesized that treatment with antidepressant medication would be associated with changes in functional coupling of the amygdala. To test this hypothesis we estimated the associations between task-related activity in the right or left amygdalae during perceptual processing of sad faces and all other voxels in functional magnetic resonance images (fMRI) of the brain acquired in a factorially designed study. Nineteen patients with depression were scanned twice during an implicit facial affect processing task, once at baseline and once following 8 weeks treatment with fluoxetine $20 \mathrm{mg} /$ day. Nineteen healthy volunteers were also scanned twice during performance of the same task, at baseline and 8 weeks later, but did not receive antidepressant treatment. This design allowed us to test for a main effect of group (patients $v s$ controls) on time-related change in right and left amygdalae coupling, that might be indicative of the effects of antidepressant treatment, as well as group-by-side interactions that might be indicative of lateralized effects of antidepressant treatment on amygdala coupling. Time-related change here refers to the difference in functional coupling of the amygdala measured at week 0 and at week 8. Different aspects of these data, based on multiple univariate analysis, have been previously reported by $\mathrm{Fu}$ et al (2004) and Chen et al (2007).

\section{MATERIALS AND METHODS}

\section{Participants}

Nineteen patients meeting DSM-IV criteria for major depressive disorder (American Psychiatric Association, 1994), as operationalized by the Structured Clinical Interview for DSM-IV Axis 1 Disorders (First et al, 1995), were recruited through local newspaper advertisements: mean age $\pm S D=43.3 \pm 8.6$ years; 13 females, 6 males. Inclusion criteria were an acute episode of major depressive disorder of the unipolar subtype and a score of at least 18 on the 17-item Hamilton Rating Scale for Depression (HAM-D; Hamilton, 1960). Exclusion criteria were a history of neurological trauma resulting in loss of consciousness, current neurological disorder, current comorbid Axis 1 disorder (including bipolar disorder and anxiety disorder), or a history of substance abuse within 2 months of study participation. All patients were free of psychotropic medication for a minimum of 4 weeks at recruitment.

Nineteen healthy comparison subjects were recruited by advertisement from the local community and matched to the patients in terms of mean age and sex: mean age \pm $\mathrm{SD}=42.8 \pm 6.7$ years; 11 females, 8 males. These comparison subjects all had HAM-D scores of less than 8 and no history of any psychiatric disorder, neurological disorder, or head injury resulting in a loss of consciousness.

All participants provided written, informed consent. The project was approved by the Ethics Research Committee, Institute of Psychiatry, London, England.

\section{Fluoxetine Administration and Clinical Assessment Protocol}

Patients received oral antidepressant treatment with fluoxetine hydrochloride, a selective serotonin reuptake inhibitor, administered in a single daily dose of $20 \mathrm{mg} / \mathrm{day}$, starting as soon as possible after the baseline clinical and MRI assessments and continuing until their completion of the study protocol 8 weeks later. For the duration of their participation in the study, the patients were assessed clinically every 2 weeks by a psychiatrist (CF) and depressive symptoms were serially rated using HAM-D. All patients recruited into the study were able to complete the protocol satisfactorily. Mean baseline symptom score was in the moderate-severe range, HAM-D $=21.3 \pm 2.4$ (SD), and was reduced by approximately $56 \%$ over the course of treatment; HAM-D at week $8=9.3 \pm 5.8$.

\section{Implicit Sad Facial Affect Processing Task}

We used an event-related fMRI paradigm with wellestablished stimuli to activate brain systems implicated in incidental processing of affectively valent faces or emotional expressions. Ten faces from a standard series of facial expressions of sadness were morphed to represent low, medium, and high intensities of sadness. Each facial stimulus was presented twice at each intensity of sadness (60 faces in total) interspersed with 12 baseline trials (crosshair fixation) in random order. For each facial trial, subjects were asked to indicate the gender of the face by lateral movement of a joystick. Total duration of the experiment was $360 \mathrm{~s}$; see Fu et al (2004) for further details on paradigm design and factorial analysis of differential activation in response to different intensities of facial affect.

\section{Functional MRI Data Acquisition}

Gradient-echo single-shot echoplanar imaging (EPI) was used to acquire $\mathrm{T} 2{ }^{*}$-weighted MR image volumes using a General Electric IGE LX System operating at $1.5 \mathrm{~T}$ at the 
Maudsley Hospital, London, UK. During an implicit facial affect processing task (detailed above), we acquired 180 3D image volumes, each comprising 16 noncontiguous axial sections parallel to the intercommissural plane, with the following parameters: $\mathrm{TR}=2000 \mathrm{~ms}$; $\mathrm{TE}=40 \mathrm{~ms}$; section thickness $=7 \mathrm{~mm}$; section skip $=0.7 \mathrm{~mm}$; and in-plane resolution $=3 \mathrm{~mm}$.

\section{Connectivity Analysis}

Connectivity analysis in functional neuroimaging has been influentially subdivided into two general classes: functional connectivity, which measures the statistical association between dynamic activity at two anatomically distinct sites in the brain; and effective connectivity, which measures the directional influence that one region exerts on activity in other regions (Friston, 1994; Friston et al, 1997). Subsequent statistical models employed in testing for effective connectivity among brain areas include structural equation modeling, causal dynamic modeling, and autoregressive modeling (Friston et al, 2003; McIntosh et al, 1994; Goebel et al, 2003). These models accommodate more complex procedures such as modeling nonlinear effects, lagged effects, or estimating several multiple regression analyses simultaneously.

We used a simple regression analysis to measure the association between the amygdala and other brain regions on a voxel by voxel basis. As summarized earlier, the connections between the amygdala and many other brain regions likely perform an important role in affect processing. Based on this evidence, we were interested in estimating the strength of functional coupling between the amygdala and other brain regions before and after antidepressant treatment. In brief, we used a linear regression model to estimate whole brain maps of functional coupling between right and left amygdalae activity - represented by regional mean time series individually extracted from the amygdala bilaterally - and all other voxels in the images. For each subject, the maps of right and left amygdalae coupling at baseline were subtracted from the maps of amygdala coupling at week 8, generating maps of the timerelated differences in right and left amygdalae coupling. These data were then treated as dependent variables in a two-way analysis of variance (ANOVA) model comprising group (patients $v s$ controls as a between-subject factor), side (left or right amygdala as a within-subject factor), and the group-by-side interaction. This model was fit identically to the coupling difference measures at each intracerebral voxel and the factors were tested for significance by a permutation test. This simple regression model provides a procedure for characterizing the strength of functional coupling between the seed region (amygdala) and other brain regions on a whole brain basis (Heinz et al, 2005). However, it cannot be used for testing a model of directional interactions among multiple brain areas guided by their known anatomical connectivity. This general strategy is described below in greater detail.

\section{Preprocessing}

Each individual fMRI dataset was preprocessed to correct for effects of head movement by image realignment and differences between slices in timing of data acquisition before affine and nonlinear spatial normalization to the Montreal Neurological Institute (MNI) EPI template image. No spatial smoothing was applied to the time series volumes. These operations were implemented using SPM2 software (http://www.fil.ion.ucl.ac.uk/spm2.html).

\section{Regional Mean Time Series Extraction from the Left and Right Amygdala}

We used the Automated Anatomical Labeling template image (AAL; Tzourio-Mazoyer et al, 2002) to define voxels in each fMRI dataset that represented the right and left amygdalae regions. Two regional mean time series were estimated for each individual by averaging the fMRI time series over all voxels in the right or left amygdala, as described previously by Salvador and colleagues (Salvador et al, 2005). Each regional mean time series was further corrected for the effects of head movement, using the statistical software R (http://www.r-project.org/), by regression on the time series of translations and rotations of the head estimated in the preprocessing step of image realignment. The residuals of these regressions constituted a new pair of regional mean time series that were used in all subsequent analysis.

\section{Functional Coupling}

For whole brain mapping of amygdala coupling, the motion corrected time series representing right and left amygdalae were separately regressed on all fMRI time series in each individual's dataset (without prior convolution by a model of the hemodynamic response function). The design matrix specified for this regression analysis also included the time series of translations and rotations of the subject's head (estimated during preprocessing) as nuisance covariates. This resulted in two maps of the regression coefficients for the effect of (right and left) amygdala activity on all other brain regions for each participant in the study. These were averaged over all participants within each group to produce maps of group mean amygdala coupling which were thresholded at $\left|t_{75}\right|>3.69, P<0.001$ (uncorrected) to represent the pattern of amygdala coupling on average over both time points (week 0 and week 8).

\section{Analysis of Variance and Significance Testing}

To identify locations of significant group, side, and groupby-side effects on changes in amygdala coupling over time from week 0 to week 8 , we subtracted the subject-specific coupling maps at week 0 from those of week 8 and performed a $2 \times 2$ mixed effects ANOVA using the difference of amygdala coupling between week 8 and week 0 as the dependent variables. This model was specified to include a main effect of group (with 2 levels, depressed patients, and healthy volunteers; an indicator of antidepressant effects regardless of side), a main effect of side (with 2 levels, left, and right), and a group-by-side interaction (an indicator of lateralized antidepressant effects on amygdala coupling).

The model was fit at each intracerebral voxel in standard space and statistical significance of each effect was tested by a cluster-level permutation test. Briefly, this involved 
applying a preliminary probability threshold $(P<0.05)$ to the corresponding voxel statistic maps and setting all subthreshold voxels to 0 , thus creating a set of suprathreshold voxel clusters that were spatially contiguous in three dimensions. The sum of the suprathreshold voxel statistics, or cluster statistic mass $\mathrm{M}$, was tested by means of a permutation test with cluster-wise probability of a type I error $P<0.005$. At these thresholds, we expect less than 1 false-positive cluster per map (Suckling and Bullmore, 2004; Bullmore et al, 1999).

We also fitted another $2 \times 2$ mixed effects ANOVA model comprising a main effect of group, a main effect of time, and the group $\times$ time interaction, with right or left amygdala coupling separately treated as the dependent variable. Factorial effects on right or left amygdala coupling were tested by a cluster-level permutation test as described above; see Supplementary Material and Supplementary Figure S1.

Finally, we made between-group comparisons, by independent $t$-tests at each voxel, to highlight differences between depressed patients and control subjects in right or left amygdala coupling at week 0 and at week 8; see Supplementary Material and Supplementary Figure S2.

\section{RESULTS}

\section{Group Maps of Mean Amygdala Coupling}

As shown in Figure 1, right and left amygdalae were positively coupled with the medial temporal lobes and ventral occipital cortex bilaterally. Amygdala was also negatively coupled with the anterior cingulate cortex. Broadly similar patterns of amygdala coupling were found for both healthy volunteers and patients with depression on average over both time points (week 0 and week 8). However, betweengroup comparisons (Supplementary Figure S2) showed that functional coupling of the left and right amygdalae was decreased in depressed patients compared to matched healthy controls at baseline in hippocampus, bilateral amygdala, putamen, insula, parahippocampal gyrus, inferior, middle, and superior temporal cortices, and inferior and middle frontal cortices. No regions showed greater functional coupling of the bilateral amygdala in depressed patients compared to healthy controls at baseline. There was no significant difference between depressed and control groups in functional coupling of the amygdala after treatment, suggesting that antidepressant treatment normalizes functional coupling of the amygdala in patients.

\section{Factorial Analysis of Changes in Amygdala Coupling}

There was evidence for a main effect of group on timerelated changes in amygdala coupling; see Figure 2. On average over both sides, patients with depression had a significantly greater time-related increase in amygdala coupling with right prefrontal cortex (approximate Brodmann areas (BA) 46, 45, 48), anterior cingulate cortex (BA 32), insula, thalamus, caudate nucleus, and putamen; see Table 1 for anatomical details. Since the patients were receiving antidepressant medication over the 8 -week period between baseline and final imaging sessions, this result indicates that antidepressant treatment may increase the

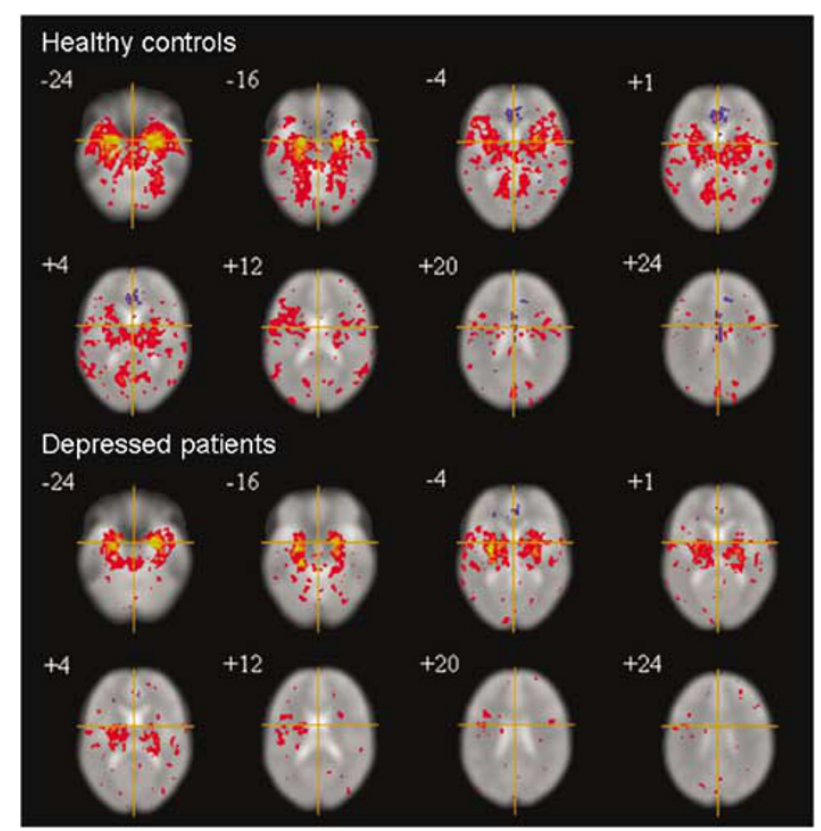

Figure I Group mean maps of functional coupling between amygdala and other brain regions in healthy volunteers and patients with depression. The figure shows the overall pattern of functional coupling of the amygdala in control or patient groups on average over both time points and both sides. The red voxels indicate the anatomical locations (eg medial temporal and ventral occipital cortices) which demonstrate positive functional coupling with the amygdala. The yellow voxels within these clusters represent peak values. The blue voxels indicate the anatomical locations (eg anterior cingulate and midcingulate cortex) which show negative functional coupling with the amygdala. The purple voxels within these clusters represent peak values. A probability threshold $\left(\left|t_{75}\right|>3.69\right.$, $P<0.00$ I (uncorrected)) is applied to all maps where subthresholded voxels are set to zero. All maps are presented in the Talairach standard space; the crosshair indicates the origin of the $x$ and $y$ dimensions and the $z$ dimension is shown numerically; the right side of each map corresponds to the right side of the brain.

functional coupling between the amygdala bilaterally and prefrontal, anterior cingulate, and subcortical regions.

We found no evidence for a significant main effect of side, indicating that time-related changes in amygdala coupling were not lateralized on average over both groups.

However, we did find evidence for a significant group-byside interaction located in left medial and lateral temporal cortex, left ventral occipital and inferior parietal cortex, left posterior cingulate cortex, right prefrontal cortex, cerebellum, insula, and putamen; see Figure 2 and Table 1 for anatomical details. Post hoc analysis demonstrated that time-related changes in left amygdala coupling with these regions were consistently greater than time-related changes in right amygdala coupling in the patients with depression (left cerebellum: $t_{18}=2.98, P=0.008$; insula, hippocampus, putamen, left temporal and left occipital cortices, left supramarginal, left postcentral, and left inferior parietal gyri: $t_{18}=3.47, P=0.003$; right middle frontal and right inferior frontal gyri, and pregenual anterior cingulate cortex: $t_{18}=4.09, P=0.001$; posterior cingulate, right superior parietal gyrus, and right calcarine gyrus: $t_{18}=$ 3.94, $P=0.001$; see Table 1); whereas time-related changes in left amygdala coupling were consistently less than timerelated changes in right amygdala coupling in the healthy 

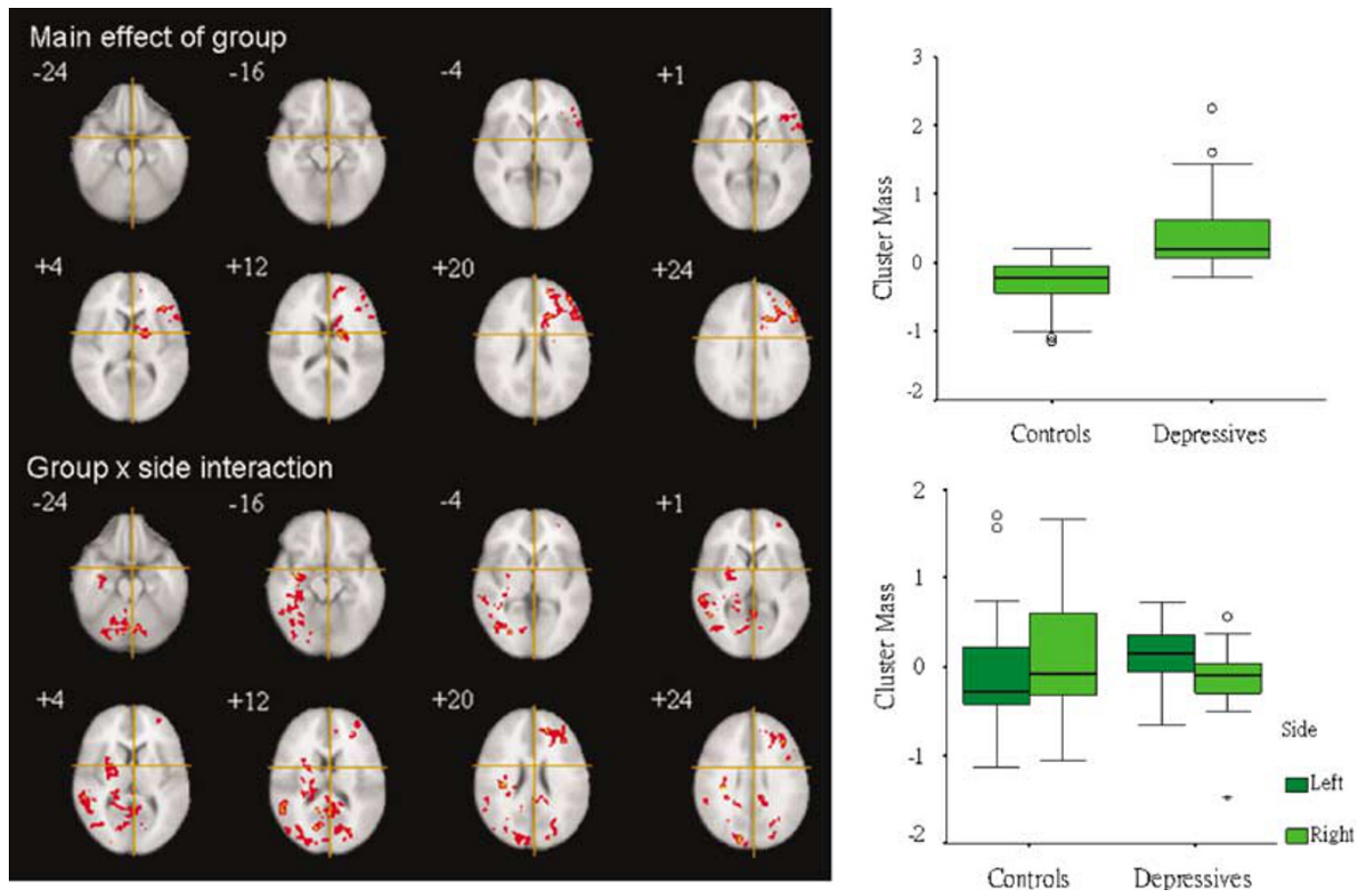

Figure 2 Factorial effects on time-related change in functional coupling of the amygdala. Left panel: The first two rows show the anatomical locations of a main effect of group on time-related change in amygdala coupling. A significantly greater time-related increase in functional coupling of bilateral amygdala to these regions (eg right prefrontal and anterior cingulate cortex) was shown in depressed patients treated with antidepressant medication over the course of 8 weeks. The last two rows show the anatomical locations of a significant group-by-side interaction. These regions show that time-related changes in functional coupling of the amygdala are significantly different between left and right amygdala in both groups. After antidepressant treatment, time-related change in left amygdala coupling with these regions was greater than time-related change in right amygdala coupling, in depressed group. All maps are presented in the Talairach standard space; the crosshair indicates the origin of the $x$ and $y$ dimensions and the $z$ dimension is shown numerically; the right side of each map corresponds to the right side of the brain. Right panel: The boxplot on the top illustrates the main effect of group. The boxplot on the bottom illustrates the group-by-side interaction. Cluster mass represents time-related change in amygdala coupling (eg a positive value represents increased amygdala coupling over the 8-week period).

volunteers (left cerebellum: $t_{18}=-3.45, P=0.003$; insula, hippocampus, putamen, left temporal and left occipital cortices, left supramarginal, left postcentral, and left inferior parietal gyri: $t_{18}=-2.85, P=0.011$; right middle frontal and right inferior frontal gyri, and pregenual anterior cingulate cortex: $t_{18}=-2.71, P=0.014$; posterior cingulate, right superior parietal gyrus, and right calcarine gyrus: $t_{18}=$ $-3.34, P=0.004$; see Table 1 ).

In Supplementary Material, we report the results of a second ANOVA model comprising a main effect of group, a main effect of time, and the group $\times$ time interaction, with right or left amygdala coupling separately treated as the dependent variable. The maps of the group $\times$ time interaction confirm that the right prefrontal cortex is the main locus of a group difference in time-related change of left amygdala coupling. There was no significant group $\times$ time effect on coupling of right amygdala. In Supplementary Figure S1, we illustrate the compatibility of this result with the one previously reported by fitting an ANOVA model comprising a main effect of group, a main effect of side (right or left amygdala), and the group $\times$ side interaction, with time-related difference in right or left amygdala coupling as the dependent variable.

Results from both models are consistent with the hypothesis that antidepressant treatment was associated with significantly increased coupling between right frontal regions and (specifically) left amygdala.

\section{DISCUSSION}

There are three main findings from this analysis: (1) in both depressed patients and healthy volunteers, and on average over both time points and both sides, the amygdala was positively coupled with medial temporal and ventral occipital regions and negatively coupled with the anterior cingulate cortex; (2) depressed patients treated with antidepressant medication over the course of 8 weeks demonstrated a significant time-related increase in functional coupling between the amygdala and right prefrontal and anterior cingulate cortex; (3) time-related increases in amygdala coupling in the patient group were significantly greater for the frontal and posterior connections of left amygdala compared to right amygdala. We discuss each of these results in turn.

\section{Positive and Negative Functional Coupling of the Amygdala}

The pattern of functional coupling represented by the group mean maps (Figure 1) is compatible with the known anatomical connections of the amygdala. Positive coupling indicates that amygdala activity is associated with increased activity in medial temporal and ventral occipital regions which could reflect the known modulating feedback connections of the amygdala to ventral occipital regions 
Table I Anatomical Locations of the Main Effect of Group and Group-by-Side Interaction on Time-Related Change in Functional Coupling of the Right and Left Amygdala in Depressed Patients and Healthy Volunteers

\begin{tabular}{|c|c|c|c|c|c|}
\hline & \multirow[t]{2}{*}{ BA } & \multicolumn{3}{|c|}{ MNI coordinates } & \multirow[t]{2}{*}{$\begin{array}{c}\text { Statistics } \\
\text { (F) }\end{array}$} \\
\hline & & $x$ & $y$ & $\mathbf{z}$ & \\
\hline \multicolumn{6}{|l|}{ Main effect of group } \\
\hline \multirow[t]{2}{*}{ Middle frontal gyrus } & 46 & 44 & 42 & 22 & 26.89 \\
\hline & 46 & 31 & 32 & 32 & 26.89 \\
\hline \multirow[t]{2}{*}{ Inferior frontal gyrus } & 45 & 47 & 37 & I & 26.89 \\
\hline & 48 & 54 & 18 & 4 & 26.89 \\
\hline $\begin{array}{l}\text { Pregenual anterior } \\
\text { cingulate }\end{array}$ & 32 & 13 & 49 & 18 & 26.89 \\
\hline Anterior midcingulate & 32 & 15 & 22 & 29 & 26.89 \\
\hline Insula & & 39 & 30 & 7 & 26.89 \\
\hline Thalamus & & 14 & -7 & 11 & 26.89 \\
\hline Putamen & & 24 & -1 & 14 & 26.89 \\
\hline Caudate & & 15 & 14 & 20 & 26.89 \\
\hline \multicolumn{6}{|l|}{ Group-by-side interaction } \\
\hline \multirow[t]{3}{*}{ Middle frontal gyrus } & 46 & 35 & 50 & 18 & 21.65 \\
\hline & 9 & 33 & 32 & 46 & 21.65 \\
\hline & 9 & 49 & 16 & 46 & 21.65 \\
\hline $\begin{array}{l}\text { Pregenual anterior } \\
\text { cingulate }\end{array}$ & 32 & 14 & 43 & 24 & 21.65 \\
\hline Inferior frontal gyrus & 45 & 38 & 30 & 26 & 21.65 \\
\hline $\begin{array}{l}\text { Posterior cingulate } \\
\text { cortex }\end{array}$ & 23 & 11 & -39 & 34 & 26.26 \\
\hline Supramarginal gyrus & 48 & -56 & -40 & 30 & 18.06 \\
\hline Superior parietal gyrus & 7 & 27 & -52 & 59 & 26.26 \\
\hline Inferior parietal gyrus & 40 & -33 & -48 & 46 & 18.06 \\
\hline Postcentral gyrus & 2 & -34 & -39 & 59 & 18.06 \\
\hline Middle temporal gyrus & 22 & -59 & -40 & 6 & 18.06 \\
\hline Inferior temporal gyrus & 37 & -38 & -34 & -12 & 18.06 \\
\hline Parahippocampus & 37 & -30 & -31 & -12 & 18.06 \\
\hline Fusiform gyrus & 19 & -29 & -64 & -10 & 18.06 \\
\hline \multirow[t]{2}{*}{ Inferior occipital gyrus } & 37 & -41 & -67 & -9 & 18.06 \\
\hline & 19 & -26 & -78 & -6 & 18.06 \\
\hline \multirow[t]{2}{*}{ Calcarine gyrus } & 18 & -14 & -74 & 14 & 18.06 \\
\hline & 18 & 16 & -74 & 14 & 26.26 \\
\hline Superior occipital gyrus & 19 & -16 & -87 & 28 & 18.06 \\
\hline Insula & & -36 & -24 & 24 & 18.06 \\
\hline \multirow[t]{2}{*}{ Hippocampus } & & -35 & -10 & -17 & 18.06 \\
\hline & & -33 & -32 & -8 & 18.06 \\
\hline \multirow[t]{2}{*}{ Putamen } & & -29 & -9 & -6 & 18.06 \\
\hline & & -27 & -8 & 8 & 18.06 \\
\hline Cerebellum & & -20 & -59 & -29 & 20.42 \\
\hline \multicolumn{6}{|l|}{ Main effect of side } \\
\hline No significance & & & & & \\
\hline
\end{tabular}

Abbreviations: BA, Brodmann's area; MNI, Montreal Neurological Institute. involved in visual processing of affectively valent stimuli (Aggleton, 1993). Negative coupling of the amygdala to anterior cingulate cortex is compatible with prior functional neuroimaging data demonstrating negative functional connectivity between the supragenual anterior cingulate cortex and the amygdala during perception of threatening faces in healthy volunteers (Pezawas et al, 2005). The authors argued that this coupling (correlation) demonstrated a feedback circuit implicated in the extinction of negative affect (Pezawas et al, 2005). However, this study also reported positive connections between the subgenual anterior cingulate and the amygdala in contrast to our negative connections observed in the same regions (Pezawas et al, 2005). This discrepancy in the sign of coupling between amygdala and subgenual cingulate cortex might be due to the different types of emotions presented in the two experiments. During perception of threatening stimuli, positive coupling, as reported previously (Pezawas et al, 2005), might be related to an excitatory influence from the amygdala to the subgenual anterior cingulate cortex. Negative connections during perception of sad faces might suggest that amygdala activity can also have an inhibitory effect on activity in the anterior cingulate cortex. Thus the amygdala activity-induced suppression or potentiation of the anterior cingulate activity might be context dependent.

In support of this interpretation, we note that one of the most important afferent connections to medial prefrontal cortex in rodents is from the basolateral nucleus of the amygdala (Bacon et al, 1996). There is additional evidence that this ascending amygdalofrontal pathway provides not only direct excitatory ascending inputs to pyramidal neurons but also to local inhibitory interneurons in the medial prefrontal cortex (Gabbott et al, 2006). Previous data in rodents has also shown that, following experimental stimulation of the amygdala, the most frequent response is reduced activity of pyramidal neurons in medial prefrontal cortex (Garcia et al, 1999; Perez-Jaranay and Vives, 1991); and reduced prefrontal cortex activity was inversely correlated with increases in amygdala neuronal activity (Garcia et al, 1999). Thus our observations of negative amygdalocingulate coupling in human functional MRI data can be regarded as compatible with the known inhibitory effects of ascending projections from amygdala to homologous regions of medial prefrontal cortex in rats. However, we note that this interpretation assumes rather a simple relationship between the sign of the functional coupling (regression) coefficient and the postsynaptic effects of amygdala input at a cellular level; whereas the nature of the relationships between functional imaging measures of connectivity and postsynaptic effects in the underlying neuronal populations is in fact controversial (Lauritzen, 2005).

\section{Antidepressant Effects on Amygdala Coupling}

We found that the influence of the amygdala on the right middle prefrontal cortex, inferior frontal gyrus, pregenual anterior cingulate, anterior midcingulate, insula, thalamus, caudate, and putamen was increased in depressed patients over the course of 8 weeks of antidepressant treatment. In other words, following treatment, a unit change in amygdala activity was associated with greater activity of a right 
fronto-striato-thalamic system in patients. It has been argued that a limbic cortical-striato-pallido-thalamic circuit, including the amygdala, medial and orbital prefrontal cortex, striatum, pallidum, and medial thalamus, is involved in the pathophysiology of depression (Drevets, 2001). This circuit plays a central role in systems-level modulation of emotional, cognitive, and motivational activities (Drevets, 2000; Sheline, 2003). There is strong evidence from anatomical studies in primates that cortical and subcortical components of this system are interconnected and receive projections from the amygdala (Alexander et al, 1986; Russchen et al, 1985; Vogt and Pandya, 1987). The amygdala plays a key role in evaluating the emotional significance of a perceived stimulus and it sends outputs to the medial and orbital prefrontal cortex which are thought to be important for response selection and decision making (Barbas, 2000; Bechara et al, 2000, 2003; LeDoux, 2000; Vogt, 2005). Both the amygdala and the prefrontal cortex have connections with the striatum (Cavada et al, 2000; Russchen et al, 1985; Alexander et al, 1986). These pathways could be involved in goal-directed behaviors (Kalivas and Nakamura, 1999; Kringelbach and Rolls, 2004). Therefore, these regions constitute key elements of a circuit through which emotional stimuli could direct behavior toward positive goals and away from aversive ones (Kalivas and Nakamura, 1999; Robbins and Everitt, 1996; Ikemoto and Panksepp, 1999). Our data indicating increased strength of functional coupling between these regions in depressed patients treated with antidepressants is compatible with the idea that the interactions between the amygdala and frontostriato-thalamic systems may be important for appropriate emotional and motivational behaviors. Increased coupling in this context can therefore be plausibly associated with the amelioration of some depressive symptoms such as depressed mood, anhedonia, fatigue, and psychomotor dysfunction.

We also observed increased coupling between the amygdala and lateral prefrontal cortex including the middle and inferior frontal gyri. The prefrontal cortex has long been argued to play an important role in cognitive control and integrating thought and action in accordance with internal goals (Miller and Cohen, 2001). Increased coupling between the amygdala and right lateral prefrontal cortex might facilitate the prefrontal modulation of amygdala activity. Our results are compatible with the view that antidepressants may increase corticolimbic coupling and enhance the cortical regulation of abnormal limbic activation (Anand et al, 2005b; Mayberg, 2002), and with prior observations that antidepressant treatment may be associated with increased frontal cortical blood flow or activation and decreased subcortical blood flow or activation (Mayberg et al, 1999, 2000; Buchsbaum et al, 1997; Kennedy et al, 2001; Davidson et al, 2003; Fu et al, 2004; Sheline et al, 2001).

Neural connectivity in major depression has been investigated in several prior brain imaging studies (Anand et al, 2005a, b; Irwin et al, 2004; Seminowicz et al, 2004). Antidepressant drug responders differed from nonresponders in a network of lateral prefrontal cortex, subgenual anterior cingulate, orbitofrontal cortex, and hippocampus using structural equation modeling (Seminowicz et al, 2004); however, these authors did not include the amygdala in their model. Increased functional connectivity between right and left amygdalae was reported in depressed patients compared to healthy controls (Irwin et al, 2004). The authors argued that due to the lack of direct anatomical connections between two sides of the amygdala, interhemispheric communication between the amygdalae must be mediated via the frontal regions or other intervening structures. Theoretically therefore reduced frontoamygdalar connectivity in depressed patients could result in reduced hemisphere-specific frontal inputs to the ipsilateral amygdala; thereby increasing the susceptibility of both right and left amygdalae to be influenced by nonlateralized inputs in common, leading to the observed increase in interhemispheric correlation. Carriers of the short allele of 5-HT transporter promoter polymorphism, who have increased risk of depression, showed relative uncoupling of amygdalo-cingulate connectivity compared to long allele carriers (Pezawas et al, 2005).

Decreased functional connectivity (correlation) between the pregenual anterior cingulate and limbic regions including amygdala, striatum, and medial thalamus has been demonstrated in unmedicated depressed patients relative to healthy controls (Anand et al, 2005a), suggesting that patients may have decreased cortical regulation of limbic regions in response to negative stimuli (Anand et al, 2005a). In a further study by the same group, functional connectivity between the anterior cingulate cortex and limbic regions was significantly increased in patients after antidepressant treatment in data acquired in the resting state and during exposure to affectively neutral and positively valent pictures. However, in response to negatively valent pictures, corticolimbic correlations remained decreased in depressed patients (Anand et al, 2005b). Our data are broadly aligned with these results in suggesting that modulation of coupling between limbic and cortical regions may be a biological marker of antidepressant drug effects on brain function. However, clearly there are also discrepancies between our findings of enhanced coupling between the amygdala and right prefrontal cortex and anterior cingulate cortex in data acquired during exposure to negatively valent stimuli and the observation by Anand et al (2005b) that the connections of the limbic structures and the pregenual anterior cingulate remain abnormally decreased in patients following 6 weeks of treatment with sertraline. Possible reasons for this discrepancy include a longer period of antidepressant treatment in our study, and differences in data analytical procedures.

The molecular mechanisms by which antidepressant drugs might modulate amygdala connectivity are not defined by these or other neuroimaging data but we note that neurotrophic effects of antidepressants have been described (Castren, 2005; Nestler et al, 2002) and it is conceivable that drug-induced synaptic plasticity could be reflected in enhanced coupling at the systems level of neuroimaging.

\section{Lateralization of Antidepressant Effects on Amygdala Coupling}

We also found evidence that the effects of antidepressant treatment on amygdala coupling were more salient for the left amygdala than for the right, suggesting that there might 
be lateralized differences in the functional effects of amygdalo-cortical projections or the susceptibility of these projections to modulation by antidepressant drugs. Resting blood flow or metabolism of the amygdala appears to be elevated on both sides of the brain in the depressed state and the left amygdala function was also specifically increased between depressive episodes in familial depressives who were not taking antidepressant treatment (Drevets, 1999, 2003). It has been argued that the elevated activity in the left amygdala in depression may result from reduced inhibitory inputs to amygdala (Drevets, 1999), and a decrease of left amygdala activity to normal was observed after chronic antidepressant treatment (Drevets et al, 2002a). In addition, most imaging studies have reported left amygdala activation when subjects were shown unmasked emotional stimuli (Iidaka et al, 2001; Ohira et al, 2006; Levesque et al, 2003; Phan et al, 2005; Adolphs, 2002) whereas the right amygdala may be activated more in response to subliminally presented stimuli (Adolphs, 2002; Morris et al, 1998). It has been suggested that the left and right amygdalae play a differential role in processing facial expressions in collaboration with other cortical or subcortical regions, with the left amygdala being related more strongly to the bilateral prefrontal cortex, and the right to the right temporal lobe (Iidaka et al, 2001). Furthermore, an increase of the right (Levesque et al, 2003; Phan et al, 2005; Ochsner et al, 2004) and left (Ohira et al, 2006) lateral prefrontal cortex activities was observed with voluntary suppression of emotion. Taken together, these results may suggest that the left rather than right amygdala might be more sensitive to unmasked emotional stimuli, particularly with negative valence, and more associated with bilateral prefrontal cortical activity, which plays a role in the regulation or suppression of emotional response. Our data showing enhanced functional coupling following antidepressant treatment between the left amygdala and right lateral prefrontal cortex in response to unmasked negative faces appear to be compatible with these observations.

\section{Methodological Issues}

The sample size is modest and the statistical power to detect significant effects on amygdala coupling may be limited by intersubject variability. The patient group was sampled from a restricted age range (mean 43.3 years) and, pending replication, our results should not be assumed to generalize to younger or more elderly patient groups.

At the first level of analysis, we used a linear regression model to map functional coupling of the right and left amygdalae and, at the second level, we used a mixed effects ANOVA model and permutation testing to identify brain regions where there were significant effects of group or group-by-side on the strength of connections. This approach has the merit of simplicity but could be refined in future studies to discriminate the components of connectivity attributable to task-related processing (Friston et al, 1997) from those attributable to endogenous or residual activity in the amygdala. Another potential refinement would be to use more sophisticated multivariate approaches to modeling effective connectivity between multiple regions, such as structural equation modeling (Bullmore et al, 2000; McIntosh et al, 1994) and dynamic causal modeling (Friston et al, 2003), which might allow a more precise disambiguation of direct $v s$ indirect effects of amygdala activity on other cortical regions. Functional connectivity (correlation) analysis has been used to study the neural substrates of depression and antidepressant drugs (Anand et al, 2005a, b; Irwin et al, 2004). This approach embodies the notion that if the activities of two brain areas are correlated, then they are likely to be functionally connected. The association is nondirectional and signal amplitude is not taken into account. A regression analysis is employed to measure the extent to which variation of activity in one brain region is predicted by that of another region. The association can be use as a measure of functional coupling between regions (Heinz et al, 2005). A model for directional interactions among multiple brain regions derived from prior theory (eg anatomical connectivity) can be tested by effective connectivity analysis (Frackowiak et al, 2004). For example, Seminowicz et al (2004)employed structural equation modeling to examine brain interactions in the limbicfrontal circuitry in major depressions. The comparison among these studies will need to consider methodological differences such as choice of analytical methods and experiment paradigms (eg task or resting). Finally it is notable that translation of results from connectivity analysis at the systems level of functional neuroimaging to the cellular level of postsynaptic inhibition or excitation is complicated by the current lack of consensus about the interpretation of negative BOLD signal changes (Lauritzen, 2005) and progress in this area is likely to depend on further studies combining fMRI and direct electrophysiological recordings from multiple sites in animal models (Lee et al, 2003).

\section{ACKNOWLEDGEMENTS}

This study was supported by: GlaxoSmithKline Research \& Development, Cambridge, UK; the Wellcome Trust, London, UK (CF); and a Human Brain Project grant from the National Institute of Mental Health and the National Institute of Biomedical Imaging \& Bioengineering.

\section{DISCLOSURE/CONFLICTS OF INTEREST}

The authors declare that over the past two years EB has been employed half time by University of Cambridge and half time by GlaxoSmithKline plc. EMP has been employed by GlaxoSmithKline plc.

\section{REFERENCES}

Adolphs R (2002). Neural systems for recognizing emotion. Curr Opin Neurobiol 12: 169-177.

Aggleton JP (1993). The Amygdala. Oxford University Press Inc.: New York.

Alexander GE, DeLong MR, Strick PL (1986). Parallel organization of functionally segregated circuits linking basal ganglia and cortex. Ann Rev Neurosci 9: 357-381.

American Psychiatric Association (1994). Diagnostic and Statistical Manual of Mental Disorders. American Psychiatric Association: Washington, DC.

Anand A, Li Y, Wang Y, Wu J, Gao S, Bukhari L et al (2005a). Activity and connectivity of brain mood regulating circuit in depression: a functional magnetic resonance study. Biol Psychiatry 57: 1079-1088. 
Anand A, Li Y, Wang Y, Wu J, Gao S, Bukhari L et al (2005b). Antidepressant effect on connectivity of the mood-regulating circuit: an FMRI study. Neuropsychopharmacology 30: 1334-1344.

Bacon SJ, Headlam AJ, Gabbott PL, Smith AD (1996). Amygdala input to medial prefrontal cortex (mPFC) in the rat: a light and electron microscope study. Brain Res 720: 211-219.

Barbas H (2000). Connections underlying the synthesis of cognition, memory, and emotion in primate prefrontal cortices. Brain Res Bull 52: 319-330.

Barden N (2004). Implication of the hypothalamic-pituitaryadrenal axis in the physiopathology of depression. J Psychiatry Neurosci 29: 185-193.

Bechara A, Damasio H, Damasio AR (2000). Emotion, decision making and the orbitofrontal cortex. Cereb Cortex 10: 295-307.

Bechara A, Damasio H, Damasio AR (2003). Role of the amygdala in decision-making. Ann NY Acad Sci 985: 356-369.

Buchsbaum MS, Wu J, Siegel BV, Hackett E, Trenary M, Abel L et al (1997). Effect of sertraline on regional metabolic rate in patients with affective disorder. Biol Psychiatry 41: 15-22.

Bullmore E, Horwitz B, Honey G, Brammer M, Williams S, Sharma T (2000). How good is good enough in path analysis of fMRI data? Neuroimage 11: 289-301.

Bullmore ET, Suckling J, Overmeyer S, Rabe-Hesketh S, Taylor E, Brammer MJ (1999). Global, voxel, and cluster tests, by theory and permutation, for a difference between two groups of structural MR images of the brain. IEEE Trans Med Imaging 18: $32-42$.

Castren E (2005). Opinion-Is mood chemistry? Nat Rev Neurosci 6: 241-246.

Cavada C, Company T, Tejedor J, Cruz-Rizzolo RJ, Reinoso-Suarez F (2000). The anatomical connections of the macaque monkey orbitofrontal cortex. A review. Cereb Cortex 10: 220-242.

Chen CH, Ridler K, Suckling J, Williams SC, Fu CH, Merlo-Pich $\mathrm{EM}$ et al (2007). Brain imaging correlates of depressive symptom severity and predictors of symptom improvement after antidepressant treatment. Biol Psychiatry 62: 407-414.

Davidson RJ, Irwin W, Anderle MJ, Kalin NH (2003). The neural substrates of affective processing in depressed patients treated with venlafaxine. Am J Psychiatry 160: 64-75.

Drevets WC (1999). Prefrontal cortical-amygdalar metabolism in major depression. Ann NY Acad Sci 877: 614-637.

Drevets WC (2000). Neuroimaging studies of mood disorders. Biol Psychiatry 48: 813-829.

Drevets WC (2001). Neuroimaging and neuropathological studies of depression: implications for the cognitive-emotional features of mood disorders. Curr Opin Neurobiol 11: 240-249.

Drevets WC (2003). Neuroimaging abnormalities in the amygdala in mood disorders. Ann NY Acad Sci 985: 420-444.

Drevets WC, Bogers W, Raichle ME (2002a). Functional anatomical correlates of antidepressant drug treatment assessed using PET measures of regional glucose metabolism. Eur Neuropsychopharmacol 12: 527-544.

Drevets WC, Price JL, Bardgett ME, Reich T, Todd RD, Raichle ME (2002b). Glucose metabolism in the amygdala in depression: relationship to diagnostic subtype and plasma cortisol levels. Pharmacol Biochem Behav 71: 431-447.

Drevets WC, Videen TO, Price JL, Preskorn SH, Carmichael ST, Raichle ME (1992). A functional anatomical study of unipolar depression. J Neurosci 12: 3628-3641.

First MB, Spitzer RL, Gibbon M, Williams JB (1995). Structured Clinical Interview for DSM-IV Axis I Disorders. American Psychiatric Pub Inc.: New York.

Frackowiak RS, Ashburner JT, Penny WD, Zeki S, Friston KJ, Frith CD et al (2004). Human Brain Function. Academic Press: London.

Friston KJ (1994). Functional and effective connectivity in neuroimaging: a synthesis. Hum Brain Mapp 2: 56-78.
Friston KJ, Buechel C, Fink GR, Morris J, Rolls E, Dolan RJ (1997). Psychophysiological and modulatory interactions in neuroimaging. Neuroimage 6: 218-229.

Friston KJ, Harrison L, Penny W (2003). Dynamic causal modelling. Neuroimage 19: 1273-1302.

Fu CHY, Williams SCR, Cleare AJ, Brammer MJ, Walsh ND, Kim J et al (2004). Attenuation of the neural response to sad faces in major depression by antidepressant treatment: a prospective, event-related functional magnetic resonance imaging study. Arch Gen Psychiatry 61: 877-889.

Gabbott PL, Warner TA, Busby SJ (2006). Amygdala input monosynaptically innervates parvalbumin immunoreactive local circuit neurons in rat medial prefrontal cortex. Neuroscience 139: 1039-1048.

Garcia R, Vouimba RM, Baudry M, Thompson RF (1999). The amygdala modulates prefrontal cortex activity relative to conditioned fear. Nature 402: 294-296.

Ghashghaei HT, Barbas H (2002). Pathways for emotion: interactions of prefrontal and anterior temporal pathways in the amygdala of the rhesus monkey. Neuroscience 115: 1261-1279.

Goebel R, Roebroeck A, Kim DS, Formisano E (2003). Investigating directed cortical interactions in time-resolved fMRI data using vector autoregressive modeling and Granger causality mapping. Magn Reson Imaging 21: 1251-1261.

Hamilton M (1960). A rating scale for depression. J Neurol Neurosurg Psychiatry 23: 56-62.

Heinz A, Braus DF, Smolka MN, Wrase J, Puls I, Hermann D et al (2005). Amygdala-prefrontal coupling depends on a genetic variation of the serotonin transporter. Nat Neurosci 8: 20-21.

Iidaka T, Omori M, Murata T, Kosaka H, Yonekura Y, Okada T et al (2001). Neural interaction of the amygdala with the prefrontal and temporal cortices in the processing of facial expressions as revealed by fMRI. J Cogn Neurosci 13: 1035-1047.

Ikemoto S, Panksepp J (1999). The role of nucleus accumbens dopamine in motivated behavior: a unifying interpretation with special reference to reward-seeking. Brain Res Rev 31: 6-41.

Irwin W, Anderle MJ, Abercrombie HC, Schaefer SM, Kalin NH, Davidson RJ (2004). Amygdalar interhemispheric functional connectivity differs between the non-depressed and depressed human brain. Neuroimage 21: 674-686.

Kalivas PW, Nakamura M (1999). Neural systems for behavioral activation and reward. Curr Opin in Neurobiol 9: 223-227.

Kennedy SH, Evans KR, Kruger S, Mayberg HS, Meyer JH, McCann $S$ et al (2001). Changes in regional brain glucose metabolism measured with positron emission tomography after paroxetine treatment of major depression. Am J Psychiatry 158: 899-905.

Kringelbach ML, Rolls ET (2004). The functional neuroanatomy of the human orbitofrontal cortex: evidence from neuroimaging and neuropsychology. Prog Neurobiol 72: 341-372.

LaBar KS, Cabeza R (2006). Cognitive neuroscience of emotional memory. Nat Rev Neurosci 7: 54-64.

Lauritzen M (2005). Reading vascular changes in brain imaging: is dendritic calcium the key? Nat Rev Neurosci 6: 77-85.

LeDoux JE (2000). Emotion circuits in the brain. Annu Rev Neurosci 23: 155-184.

Lee L, Harrison LM, Mechelli A (2003). The Functional Brain Connectivity Workshop: report and commentary. Network 14: $\mathrm{R} 1-\mathrm{R} 15$.

Levesque J, Eugene F, Joanette Y, Paquette V, Mensour B, Beaudoin G et al (2003). Neural circuitry underlying voluntary suppression of sadness. Biol Psychiatry 53: 502-510.

Lopez JF, Akil H, Watson SJ (1999). Neural circuits mediating stress. Biol Psychiatry 46: 1461-1471.

Mayberg HS (2002). Modulating limbic-cortical circuits in depression: targets of antidepressant treatments. Semin Clin Neuropsychiatry 7: 255-268.

Mayberg HS, Brannan SK, Tekell JL, Silva JA, Mahurin RK, McGinnis S et al (2000). Regional metabolic effects of fluoxetine 
in major depression: serial changes and relationship to clinical response. Biol Psychiatry 48: 830-843.

Mayberg HS, Liotti M, Brannan SK, McGinnis S, Mahurin RK, Jerabek PA et al (1999). Reciprocal limbic-cortical function and negative mood: converging PET findings in depression and normal sadness. Am J Psychiatry 156: 675-682.

McIntosh AR, Grady CL, Ungerleider LG, Haxby JV, Rapoport SI, Horwitz B (1994). Network analysis of cortical visual pathways mapped with PET. J Neurosci 14: 655-666.

Miller EK, Cohen JD (2001). An integrative theory of prefrontal cortex function. Annu Rev Neurosci 24: 167-202.

Morris JS, Ohman A, Dolan RJ (1998). Conscious and unconscious emotional learning in the human amygdala. Nature 393: 467-470.

Nestler EJ, Barrot M, DiLeone RJ, Eisch AJ, Gold SJ, Monteggia LM (2002). Neurobiology of depression. Neuron 34: 13-25.

Ochsner KN, Ray RD, Cooper JC, Robertson ER, Chopra S, Gabrieli JDE et al (2004). For better or for worse: neural systems supporting the cognitive down- and up-regulation of negative emotion. Neuroimage 23: 483-499.

Ohira H, Nomura M, Ichikawa N, Isowa T, Iidaka T, Sato A et al (2006). Association of neural and physiological responses during voluntary emotion suppression. Neuroimage 29: 721-733.

Perez-Jaranay JM, Vives F (1991). Electrophysiological study of the response of medial prefrontal cortex neurons to stimulation of the basolateral nucleus of the amygdala in the rat. Brain Res 564: 97-101.

Pezawas L, Meyer-Lindenberg A, Drabant EM, Verchinski BA, Munoz KE, Kolachana BS et al (2005). 5-HTTLPR polymorphism impacts human cingulate-amygdala interactions: a genetic susceptibility mechanism for depression. Nat Neurosci 8: 828-834.

Phan KL, Fitzgerald DA, Nathan PJ, Moore GJ, Uhde TW, Tancer ME (2005). Neural substrates for voluntary suppression of negative affect: a functional magnetic resonance imaging study. Biol Psychiatry 57: 210-219.

Potter WZ, Scheinin M, Golden RN, Rudorfer MV, Cowdry RW, Calil HM et al (1985). Selective antidepressants and cerebrospinal fluid. Lack of specificity on norepinephrine and serotonin metabolites. Arch Gen Psychiatry 42: 1171-1177.
Robbins TW, Everitt BJ (1996). Neurobehavioural mechanisms of reward and motivation. Curr Opin Neurobiol 6: 228-236.

Russchen FT, Bakst I, Amaral DG, Price JL (1985). The amygdalostriatal projections in the monkey. An anterograde tracing study. Brain Res 329: 241-257.

Salvador R, Suckling J, Coleman MR, Pickard JD, Menon D, Bullmore E (2005). Neurophysiological architecture of functional magnetic resonance images of human brain. Cereb Cortex 15: $1332-1342$.

Seminowicz DA, Mayberg HS, McIntosh AR, Goldapple K, Kennedy S, Segal Z et al (2004). Limbic-frontal circuitry in major depression: a path modeling metanalysis. Neuroimage 22: 409-418.

Sheline YI (2003). Neuroimaging studies of mood disorder effects on the brain. Biol Psychiatry 54: 338-352.

Sheline YI, Barch DM, Donnelly JM, Ollinger JM, Snyder AZ, Mintun MA (2001). Increased amygdala response to masked emotional faces in depressed subjects resolves with antidepressant treatment: an fMRI study. Biol Psychiatry 50: 651-658.

Siegle GJ, Steinhauer SR, Thase ME, Stenger VA, Carter CS (2002). Can't shake that feeling: event-related fMRI assessment of sustained amygdala activity in response to emotional information in depressed individuals. Biol Psychiatry 51: 693-707.

Suckling J, Bullmore E (2004). Permutation tests for factorially designed neuroimaging experiments. Hum Brain Mapp 22: 193-205.

Tzourio-Mazoyer N, Landeau B, Papathanassiou D, Crivello F, Etard O, Delacroix N et al. (2002). Automated anatomical labelling of activations in SPM using a macroscopic anatomical parcellation of the MNI MRI single-subject brain. NeuroImage 15: 273-289.

Vogt BA (2005). Pain and emotion interactions in subregions of the cingulate gyrus. Nat Rev Neurosci 6: 533-544.

Vogt BA, Pandya DN (1987). Cingulate cortex of the rhesus monkey: II. Cortical afferents. J Comp Neurol 262: 271-289.

Wong ML, Kling MA, Munson PJ, Listwak S, Licinio J, Prolo P et al (2000). Pronounced and sustained central hypernoradrenergic function in major depression with melancholic features: relation to hypercortisolism and corticotropin-releasing hormone. Proc Natl Acad Sci USA 97: 325-330.

Supplementary Information accompanies the paper on the Neuropsychopharmacology website (http://www.nature.com/npp) 